\title{
The role of the CNOT1 subunit of the CCR4-NOT complex in mRNA deadenylation and cell viability
}

\author{
Kentaro Ito ${ }^{1}$, Akinori Takahashi ${ }^{1}$, Masahiro Morita ${ }^{1}$, Toru Suzuki ${ }^{1}$, Tadashi Yamamoto ${ }^{1,2} \bowtie$ \\ 1 Division of Oncology, Department of Cancer Biology, Institute of Medical Science, University of Tokyo, Tokyo 108-8639, Japan \\ ${ }^{2}$ Cell Signal Unit, Okinawa Institute of Science and Technology, 1919-1, Okinawa 904-0412, Japan \\ \ Correspondence: tyamamot@ims.u-tokyo.ac.jp \\ Received August 9, 2011 Accepted August 30, 2011
}

\section{ABSTRACT}

The human CCR4-NOT deadenylase complex consists of at least nine enzymatic and non-enzymatic subunits. Accumulating evidence suggests that the non-enzymatic subunits are involved in the regulation of MRNA deadenylation, although their precise roles remain to be established. In this study, we addressed the function of the CNOT1 subunit by depleting its expression in HeLa cells. Flow cytometric analysis revealed that the sub $G_{1}$ fraction was increased in CNOT1-depleted cells. Virtually, the same level of the sub $G_{1}$ fraction was seen when cells were treated with a mixture of siRNAs targeted against all enzymatic subunits, suggesting that CNOT1 depletion induces apoptosis by destroying the CCR4-NOT-associated deadenylase activity. Further analysis revealed that CNOT1 depletion leads to a reduction in the amount of other CCR4-NOT subunits. Importantly, the specific activity of the CNOT6L immunoprecipitates-associated deadenylase from CNOT1-depleted cells was less than that from control cells. The formation of P-bodies, where mRNA decay is reported to take place, was largely suppressed in CNOT1-depleted cells. Therefore, CNOT1 has an important role in exhibiting enzymatic activity of the CCR4-NOT complex, and thus is critical in control of mRNA deadenylation and mRNA decay. We further showed that CNOT1 depletion enhanced CHOP mRNA levels and activated caspase-4, which is associated with endoplasmic reticulum ER stress-induced apoptosis. Taken together, CNOT1 depletion structurally and functionally deteriorates the CCR4-NOT complex and induces stabilization of mRNAs, which results in the increment of translation causing ER stress-mediated apoptosis. We conclude that CNOT1 contributes to cell viability by securing the activity of the CCR4-NOT deadenylase.

KEYWORDS deadenylation, CCR4-NOT, small interfering RNA, P-bodies, apoptosis

\section{INTRODUCTION}

The accurate control of gene expression is essential for the normal growth and function of cells, and their dysregulation leads to a variety of disorders, including cancer, diabetes, and neuronal defects. Among the controls of gene expression, posttranscriptional mechanisms play a large role. The regulation of mRNA stability, in particular, is vitally important because it determines the availability of mRNAs for translation. Most mRNAs have a poly $(A)$ tail at their $3^{\prime}$ ends, which plays an important role in the regulation of translation and the degradation of mRNAs. Once poly(A) tail shortening takes place, mRNA degradation proceeds from either the $5^{\prime}$ or the $3^{\prime}$ end (Garneau et al., 2007).

The CCR4-NOT complex is the major deadenylase that is conserved from yeast to human (Collart and Timmers, 2004, Bartlam and Yamamoto, 2010). The complex consists of catalytic subunits termed CNOT6 and CNOT6L, which belong to the exonuclease-endonuclease-phosphatase (EEP) family, and CNOT7 and CNOT8, which belong to DEDD (Asp-GluAsp-Asp) family (Bartlam and Yamamoto, 2010). In addition, this complex contains non-catalytic subunits that include CNOT1, CNOT2, CNOT3, CNOT9 and CNOT10 in mammals. Accumulating data shows that each catalytic or non-catalytic subunit plays a distinct role in different species 
and tissues. For example, short-hairpin RNA (shRNA)mediated depletion of CNOT6L in NIH3T3 cells induces $\mathrm{G}_{1}$ arrest, but CNOT6 depletion has little effect on cell growth (Morita et al., 2007). In Drosophila, CNOT7 is proposed to be a major catalytic subunit of the CCR4-NOT deadenylase complex (Temme et al, 2010). Interestingly however, CNOT7deficient mice show defect in spermatogenesis with no other defects (Berthet et al., 2004, Nakamura et al., 2004), suggesting that other deadenylase subunits catalyze deadenylation in most tissues.

Despite the increased understanding of the structural properties of the subunits of the CCR4-NOT complex (Graces et al., 2009; Bartlam and Yamamoto, 2010; Wang et al., 2010), it is not clear how each subunit is structurally integrated into the complex. The role of each subunit in the complex is also far from clear. The subunits of the CCR4-NOT complex show distinct tissue distributions (Chen et al., 2011), suggesting that the composition of the CCR4-NOT complex differs among tissues, which may reflect tissue-specific functions of this complex. Some subunits are thought to participate in transcriptional regulation. For example, CNOT1 has been shown to interact with nuclear receptors (Winkler et al., 2006), and CNOT3 is thought to be involved in chromatin remodeling, thereby playing a role in a transcriptional mechanism (Neely et al., 2010). Recent evidence also suggests that CNOT1, CNOT2, and CNOT3 participate in the control of deadenylase activity (Tucker et al., 2002, Temme et al., 2010). In Drosophila, microRNA (miRNA)-dependent deadenylation is suppressed by CNOT1 depletion (BehmAnsmant et al., 2006, Temme et al., 2010) and CNOT2 depletion affects the length of mRNA poly(A) tails in Drosophila (Temme et al., 2004). CNOT2 depletion in human HeLa cells increases the amount of translatable mRNAs, probably by stabilization of mRNAs through limited deadenylation (Ito et al, 2011). Slight poly(A)-tail lengthening is seen in yeast not3 mutants (Tucker et al., 2002). Furthermore, the Drosophila NOT3 subunit recruits the CCR4-NOT deadenylase to its target mRNA (Chicoine et al., 2007). Therefore, the non-catalytic subunits are thought to be involved in the regulation of the deadenylase activity of the complex. Although CNOT1 functions as a scaffold subunit of the complex, the mechanism by which it exhibits its regulatory effect on the deadenylase activity and the structural organization of the complex has not been fully established.

In this study, we addressed the function of mammalian CNOT1 by depleting it from cultured human cells using short interfering RNAs (siRNAs). We found that CNOT1 depletion destabilized some components of the CCR4-NOT complex and impaired its deadenylase activity. Furthermore, CNOT1 depletion induced apoptosis in a caspase-dependent manner. Thus, human CNOT1 is important for maintaining the deadenylase activity and structural integrity of the CCR4NOT complex, thereby affecting cell viability.

\section{RESULTS}

\section{CNOT1 depletion induces apoptotic cell death}

We previously reported that CNOT2 depletion induces endoplasmic reticulum (ER) stress and causes apoptotic cell death in a manner dependent on the ER stress-activated caspase (Ito et al., 2011). To examine further the cellular function of the other subunits of the CCR4-NOT complex, we employed siRNA-mediated gene suppression and suppressed the expression of every subunit individually or all catalytic subunits simultaneously in HeLa cells. Suppression of the expression of individual (Fig. 1A) or all catalytic subunits (Fig. 1B) was confirmed by western blotting. By analyzing the siRNA-treated cells with flow cytometry, we found that a significant population of CNOT1-depleted cells, like CNOT2-depleted cells, was present in the sub $\mathrm{G}_{1}$ fraction (Fig. 1C). Depletion of most of the other subunits also increased the proportion of the cell population in the sub $\mathrm{G}_{1}$ fraction, although less significantly as compared with CNOT1 or CNOT2 depletion (Fig. 1D). The cells in sub $\mathrm{G}_{1}$ fraction are considered to represent apoptotic cells. Indeed, cleavage of poly ADP ribose polymerase (PARP) was detected in CNOT1 siRNA-treated cells and this cleavage was prevented by administrating the pan-caspase inhibitor z-VAD-fmk (Fig. 1E), strongly supporting the hypothesis that CNOT1 depletion induces apoptotic cell death in a caspase-dependent manner. Taken together, we concluded that caspase-induced apoptosis occurred upon CNOT1 depletion, as well as upon CNOT2 depletion.

Depletion of other subunits appeared to induce apoptosis to certain degrees, though less efficiently than that induced by CNOT1 or CNOT2 depletion. Interestingly however, simultaneous depletion of the four catalytic subunits, CNOT6, CNOT6L, CNOT7 and CNOT8, induced apoptosis at a level similar to that induced by the CNOT1 or CNOT2 depletion (Fig. 1D). This result may suggest that apoptosis induced by CNOT1 or CNOT2 depletion is due to structural deterioration of the CCR-NOT complex that results in inactivation of the deadenylase activity. Immunoblot analysis revealed that the amounts of CNOT2, CNOT6L, CNOT7, and CNOT9 were decreased by CNOT1 depletion (Fig. 2A). The decrease was prevented by incubating CNOT1 siRNA-treated cells with MG132, a proteasome inhibitor (Fig. 2B), suggesting that CNOT1 is important for stabilizing the components of the CCR4-NOT complex and maintaining the complex's structural integrity.

\section{Depletion of CNOT1 decreases the deadenylase activity of the CCR4-NOT complex}

To demonstrate the roles of CNOT1 in deadenylation catalyzed by the CCR4-NOT complex, we examined the effect of CNOT1 depletion on the in vitro deadenylase activity 
A

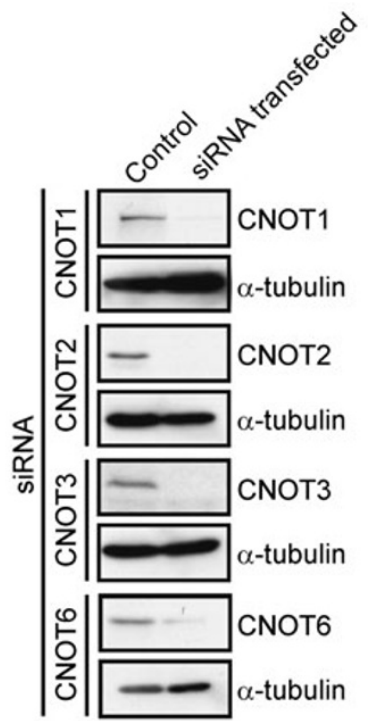

C
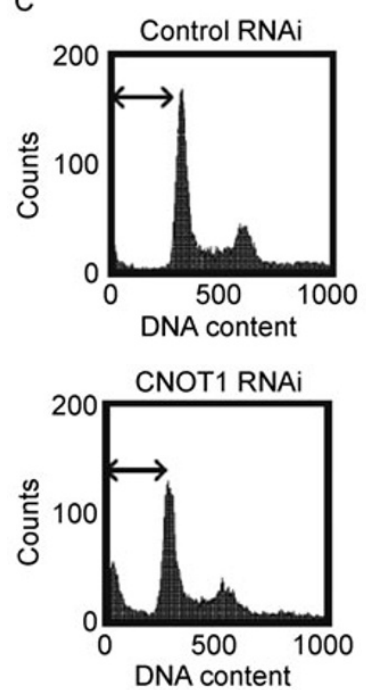

B
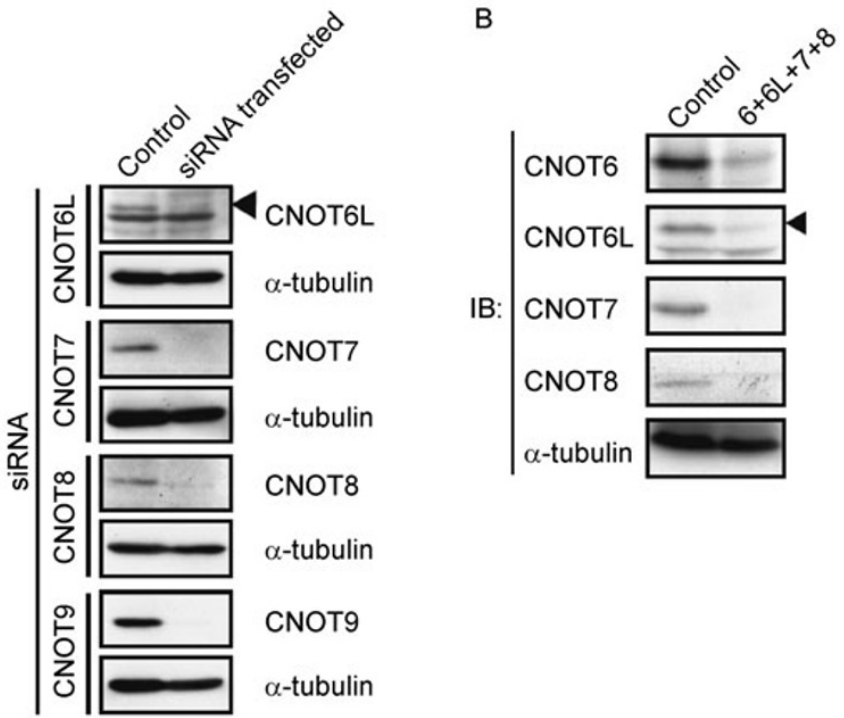

D

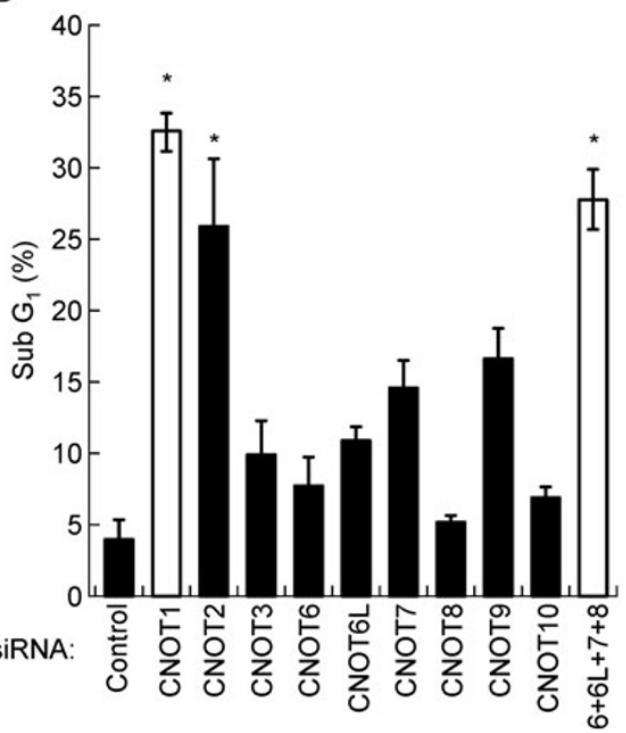

E

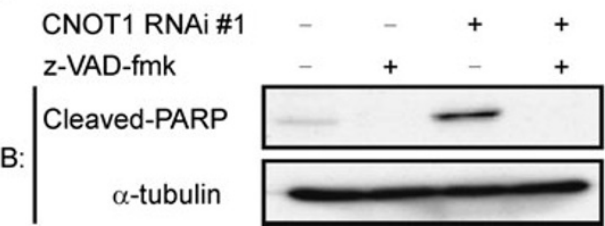

Figure 1. Depletion of CNOT1 induces cell death in a caspase-dependent manner. (A-D) HeLa cells were transfected with control siRNA or siRNAs of the CCR4-NOT subunits. Seventy-two hours after siRNA transfection, cells were harvested for the following analyses. (A and B) Immunoblot analysis of siRNAs targeting efficiency. Arrowheads indicate the CNOT6L-specific bands. $6+6 \mathrm{~L}+7+8$ : simultaneous knock-down of CNOT6, 6L, 7, and 8. (C and D) FACS analysis of apoptotic cell death. The histogram shows the DNA contents of control and CNOT1-depleted cells. The percentage of the sub $\mathrm{G}_{1}$ fraction was quantified and is shown on the right. Error bars represent the SE from three independent experiments. *: $p<0.05$, Student's $t$-test for control siRNA versus CNOT1 siRNA, CNOT2 siRNA and CNOT6, 6L, 7, and 8 mixed siRNA. (E) Immunoblot analysis of PARP cleavage. Twenty-four hours after siRNA transfection, the caspase inhibitor Z-VAD-fmk was added to the culture medium, and after another $24 \mathrm{~h}$, the cells were harvested for immunoblot analysis using an anti-cleaved PARP antibody (right). $\alpha$-tubulin blotting was included as an internal control for protein loading. 
A

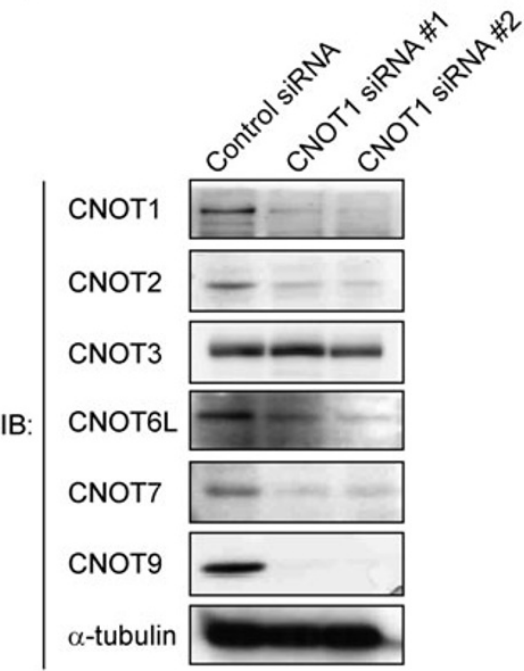

B

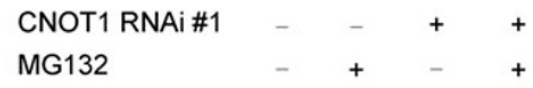

MG132

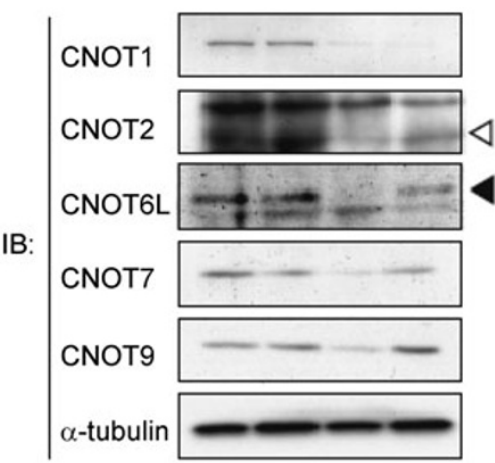

Figure 2. Depletion of CNOT1 leads to a decrease in CCR4-NOT subunits. (A) HeLa cells were transfected with control or CNOT1 siRNA. Seventy-two hours after siRNA transfection, cells were lysed and the lysates analyzed by immunoblot with the indicated antibodies. (B) Sixty-six hours after siRNA transfection, the proteasome inhibitor MG132 was added to the culture medium, and after another six hours, the cells were harvested for immunoblot analysis with the indicated antibodies. The open and closed arrowheads indicate the CNOT2- and CNOT6L-specific bands, respectively.

of the complex. Twenty-four hours after siRNA transfection, HEK293T cells were further transfected with the EGFPCNOT6L expression plasmid and the cells were incubated for an additional $24 \mathrm{~h}$. After preparation of the cell lysates, CNOT6L and associated proteins were purified using antiGFP-antibodies, and the purified proteins were subsequently subjected to an in vitro deadenylase assay in which these proteins were incubated with a poly(A) RNA. By analyzing the reaction products on a denaturing sequencing gel, we found that the anti-GFP immunoprecipitates from CNOT1-depleted cells deadenylated poly $(A)$ substrates less efficiently than the immunoprecipitates from control cells (Fig. 3A). Note that the level of exogenously expressed EGFP-CNOT6L was not affected by CNOT1 depletion (Fig. 3B). As the EGFPCNOT6L-associated deadenylase activity is impaired in the absence of CNOT1, we assumed that CNOT1 depletion reduces the deadenylase activity not only of exogenous CNOT6L, but also that of endogenous CNOT6L, as well as that of the whole complex.

\section{Depletion of CNOT1 leads to a decrease in the number of P-bodies}

Although the CCR4-NOT complex can be involved in transcriptional regulation, as evidenced by the interactions between CNOT1 and estrogen receptor $\alpha$ and retinoid $X$ receptor (RXR) (Winkler et al., 2006), this complex also plays a role in cytoplasmic mRNA decay. To substantiate the roles of CNOT1 in the nucleus and the cytoplasm, we examined the subcellular localization of CNOT1 in HeLa cells.
Immunofluorescence microscopy of cells transfected with a Flag-CNOT1 expression plasmid revealed that Flag-CNOT1 was present predominantly in the cytoplasm (Fig. 4A). Furthermore, we found Flag-CNOT1 on the RNA granules called P-bodies. As many proteins involved in mRNA decay are present in P-bodies (Sheth and Parker, 2003), CNOT1 may be involved in the control of mRNA decay. Importantly, the number of P-bodies monitored using Ago2 (Argonaute 2 protein) staining decreased upon siRNA-mediated depletion of CNOT1 expression (Fig. 4B and 4C). Note that CNOT1 depletion did not affect the expression level of Ago2 (Fig. 4D). These results suggest that CNOT1 depletion decreases the level of cytoplasmic mRNA decay.

\section{CNOT1 depletion causes ER stress-mediated cell death}

Because mRNA turnover begins with deadenylation, followed by decapping and 5' to 3' exonucleolytic decay, which occur in P-bodies (Sheth and Parker, 2003), the lack of P-body formation is indicative of limited mRNA deadenylation and limited mRNA turnover. Slow mRNA degradation could lead to an accumulation of mRNAs and, eventually, to an increase in translation. In vivo protein folding requires complex ERresident protein-folding machinery, and the overproduction of protein could result in the exhaustion of the capacity of this protein folding machinery. This exhaustion would, in turn, result in the accumulation of unfolded, aggregated proteins in the ER, inducing ER stress and, subsequently, cell death (Schroder and Kaufman, 2005). Therefore, CNOT1-depleted cells could die as a result of ER stress-induced cell death. We 
A

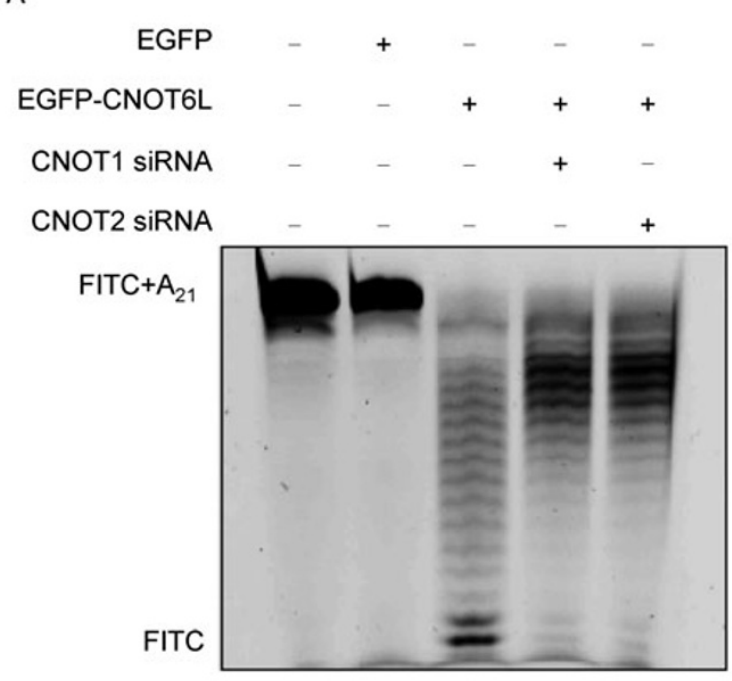

B

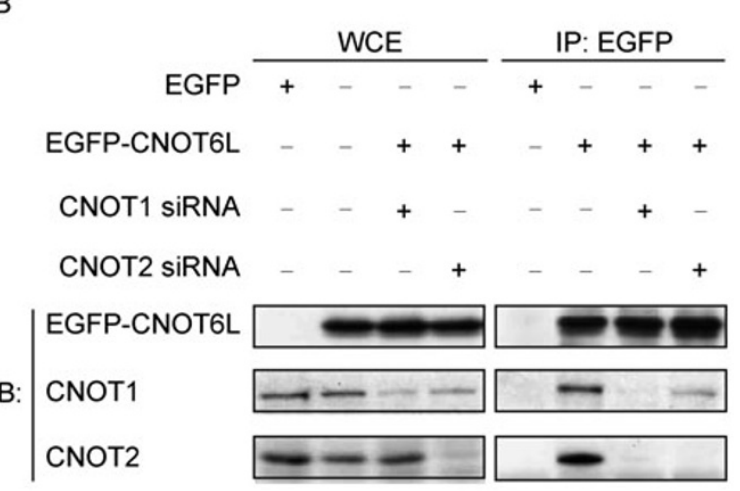

Figure 3. The deadenylase activity of the CCR4-NOT complex is reduced by depletion of CNOT1. Control and CNOT1-depleted HEK293T cells were transfected with an EGFP-CNOT6L expression plasmid and subjected to immunoprecipitation with an anti-GFP antibody. 5'-fluorescein isothiocyanate-labeled RNA substrates were incubated with the anti-GFP immunoprecipitates for three hours. The reaction products and the precipitates were analyzed on a denaturing sequence gel $(A)$ and by immunoblotting with the indicated antibodies $(B)$, respectively. The experiments were performed with CNOT2-depeleted cells as well, and the CNOT2 part in (A) and (B) was previously published (Ito et al., 2010).

examined whether caspase-4, an ortholog of murine caspase-12 that is involved in ER stress-mediated apoptotic cell death (Hitomi et al., 2004), is cleaved in CNOT1-depleted cells. Immunoblot analysis revealed that caspase-4 cleavage occurred in CNOT1 siRNA-treated HeLa cells (Fig. 5A). We further examined the transcription of CHOP/GADD153 mRNA, which occurs only after the cells are exposed to ER stress (Zinszner et al., 1998). Quantitative RT-PCR analysis showed that the amount of CHOP mRNA in CNOT1-depleted cells was about three to four times greater than that in control cells (Fig. 5B). These results suggest that CNOT1 depletion induces ER stress and mediates caspase-dependent cell death.

\section{DISCUSSION}

The CCR4-NOT complex regulates gene expression through the control of mRNA metabolism, via the complex's involvement in the events from synthesis to decay (Collart and Timmers, 2004). Accumulating evidence shows that the complex, consisting of more than nine subunits (Temme et al., 2010), is the main enzyme responsible for mRNA deadenylation (Bartlam and Yamamoto, 2010), and contributes to the regulation of mRNA stability and translation. However, despite an increasing number of studies on the CCR4-NOT complex, the role of each subunit in mRNA metabolism is far from clear. In this study, by addressing the role of CNOT1 in human cells, we had the following findings. First, CNOT1 is important for the stable expression of the other components of the complex and thus contributes to the formation of the complex. Secondly, CNOT1 positively regulates the deadenylase activity of the complex. Thirdly, human CNOT1 exists predominantly in the cytoplasm and is involved in P-body formation. Finally, CNOT1-depleted cells die as a result of ER stress-induced apoptosis, which is probably caused by protein overproduction. These results suggest that CNOT1 is important for the control of cell viability through its ability to support the structure and function of the CCR4-NOT deadenylase.

Depletion of all four enzymatic subunits of the CCR4-NOT induces apoptotic cell death; the extent of the cell death was similar to that seen upon CNOT1 or CNOT2 depletion. This result suggests that both $\mathrm{CNOT} 1$ and $\mathrm{CNOT} 2$ are relevant to the deadenylase activity of the complex, although the mechanisms by which CNOT1 and CNOT2 contribute to the formation and enzymatic activity of CCR4-NOT appear to be distinct from each other (discussed below). Because the in vitro deadenylase activity of the complex co-immunoprecipitated with exogenously expressed CNOT6L was weaker when prepared from CNOT1-depleted cells than when prepared from wild type cells, we speculate that the complex is structurally and functionally fragile if an ample amount of CNOT1 is not present. Namely, CNOT1 appears to contribute to the CCR4-NOT deadenylase activity by stabilizing the core subunits of the complex, functioning as a scaffold as previously reported (Collart, 2003). This suggests that mRNA decay in cells devoid of CNOT1 occurs slowly. Indeed, the formation of P-bodies, the sites where mRNA decay machinery degrades deadenylated mRNAs (Sheth and Parker, 2003), was suppressed in the CNOT1-depleted cells. The depletion of NOT1 in Drosophila melanogaster $\mathrm{S} 2$ cells also leads to a decrease in the number or dispersion of P-bodies (Eulalio et al., 2007). P-body formation is also suppressed in CNOT2-depleted HeLa cells probably due to 
A

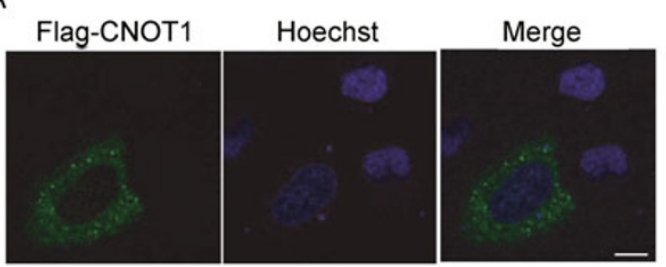

B

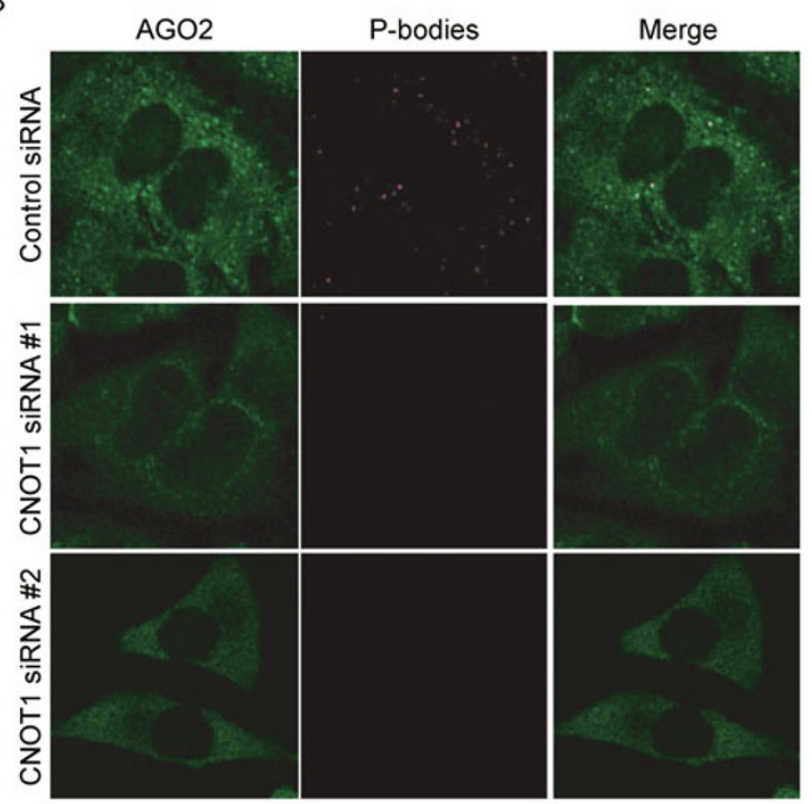

C

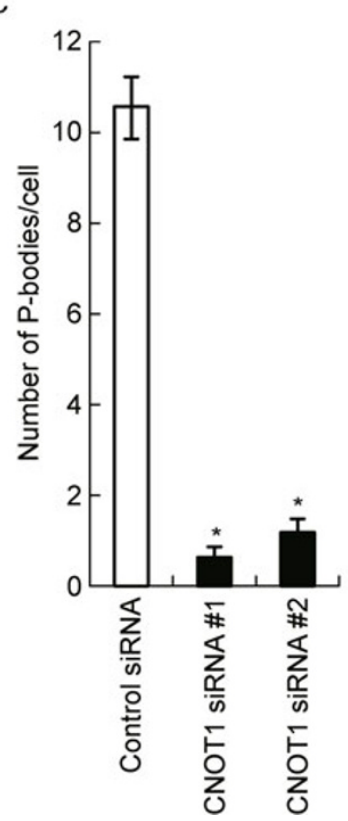

D

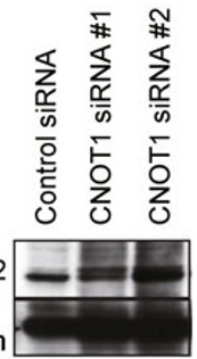

Figure 4. Depletion of CNOT1 suppresses the formation of P-bodies. (A) HeLa cells were transfected with a Flag-CNOT1 expression plasmid and fixed $24 \mathrm{~h}$ after transfection. The cells were stained with an anti-Flag antibody (green) and the expression was examined by fluorescence microscopy. (B) Forty-eight hours after control or CNOT1 siRNA transfection, the cells were stained with an anti-AGO2 antibody (green) for P-bodies (left panels). Signals were visualized by con-focal microscopy. P-body signals were extracted by Photoshop and are shown in magenta (middle panels). (C) A histogram representing the number of P-bodies shown in (B). At least 50 cells were counted per sample in four independent experiments. ${ }^{*}: p<0.05$, Student's $t$-test for control siRNA versus CNOT1 siRNA. Error bars represent the SE. (D) Forty-eight hours after CNOT1 siRNA transfection, cells were harvested and the lysates were subjected to immunoblotting with an anti-AGO2 antibody. 
impaired mRNA deadenylation (Ito et al., 2011), although the roles of CNOT1 and CNOT2 in maintaining the structural integrity of the CCR4-NOT complex appear to be distinct from each other. While CNOT1 depletion resulted in the destabilization of the other components, as we showed in this study, CNOT2 depletion has a lesser effect on the abundance of the other components except for CNOT3 (Ito et al., 2011). Interestingly, the level of the CNOT3 subunit was affected less by CNOT1 depletion than by CNOT2 depletion. We conclude that the structural integrity and enzymatic activity of the CCR4-NOT complex are controlled differently by CNOT1 and CNOT2.

Limited deadenylation leads to the stabilization of mRNAs and, thereby, an increase in the amount of translatable mRNA and the overproduction of proteins, which could result in exhaustion of the capacity of the ER-resident protein folding machinery. The unfolded proteins perturb the ER function, resulting in the activation of the unfolded protein response (UPR) (Schroder and Kaufman, 2005). One of the unfolded protein responses is the activation of PERK (protein kinase RNA-like ER kinase), which in turn induces CHOP gene expression (Harding et al., 2000). The CHOP mRNA is barely expressed under normal conditions, and its elevated expression could amplify the pro-apoptotic signal by altering the balance between Bcl-2 and Bax (McCullough et al., 2001). Therefore, we propose that apoptosis induced by CNOT1 depletion, as is the case of CNOT2 depletion, is mediated by the up-regulation of the CHOP mRNA. In case of CNOT2 depletion, cells die by apoptosis that is caused, at least in part, by protein overproduction (Ito et al., 2011). Although we have not examined the overproduction of proteins or the generation of unfolded/misfolded proteins in CNOT1depleted cells, we assumed that CNOT1-depleted cells undergo apoptosis as a result of prolonged ER stress caused by protein overproduction, as CNOT2-depleted cells do. Activation of the ER-resident caspase-4, a counterpart of murine caspase-12 that becomes activated by ER stress (Nakagawa et al., 2000), was seen upon CNOT1 depletion (this study) and CNOT2 depletion (Ito et al., 2011). Therefore, it is likely that CNOT1- and CNOT2-depleted cells undergo caspase-dependent apoptosis. In yeast, the $\Delta$ not1 mutant is lethal (Maillet et al., 2000). Although the underlying mechanism for the lethality is not clear, it is possible that cells die by ER stress-mediated apoptosis.

The CCR4-NOT complex is involved in basal deadenylation, targeting bulk mRNA (Temme et al., 2004). The complex can also participate in the regulated deadenylation of specific mRNAs such as $h s p 70$ mRNA (Temme et al., 2004) and cyclin B mRNA (Kadylova et al., 2007). As regulated deadenylation appears to occur on the unstable mRNAs (Temme et al., 2010), we speculate that CNOT1 depletion in mammals may also affect the deadenylation of a specific set of unstable mRNAs. Indeed, CNOT1 interacts with tristetraprolin (TTP), an RNA binding protein required for AU-rich element (ARE)-mediated mRNA decay, and recruits the deadenylase subunits to the ARE-containing target mRNAs (Sandler et al., 2011). Because CHOP mRNA contains ARE, theoretically, CHOP mRNA could be one of the targets of the CCR4-NOT and thus may be slowly deadenylated and stabilized in the CNOT1-depleted cells. This possibility needs to be further explored.

In the present study, we showed that CNOT1 is essential for the viability of cells because of its role in the control of deadenylation. It should be noted, however, that CNOT1 could interact with various proteins, including nuclear receptors (Winkler et al., 2006). Thereby, CNOT1 could participate in important biological processes. The biological significance of CNOT1 could be addressed by analyzing Cnot1-deficient mice. Because the CNOT1 depletion led HeLa cells to die, we assume that simple depletion of Cnot 1 would induce embryonic lethality. Future studies using conditional Cnot1-deficient mice that can undergo tissuespecific depletion of the gene will help to clarify more comprehensively the biological significance of CNOT1.

\section{MATERIALS AND METHODS}

\section{Antibodies and plasmids}

Rabbit anti-CNOT1, CNOT3, CNOT6L, CNOT7, CNOT8, and CNOT9 polyclonal antibodies were generated by immunizing rabbits (Morita et al., 2007). Anti-CNOT2 and CNOT6 monoclonal antibody was obtained from Biomatrix. Anti-PARP and anti-tubulin antibodies were from ZYMED and Sigma, respectively. Anti-GFP antibody and anticaspase-4 antibody were from MBL. Anti-human AGO2 monoclonal antibody (4B1) was from WAKO. Expression plasmid for CNOT1 (pME18S-Flag-CNOT1) and CNOT6L (pEGFP-CNOT6L) were previously described (Morita et al., 2007; Miyasaka et al., 2008).

\section{Cell culture, transfection, and inhibitors}

HeLa cells and HEK293T cells were cultured in Dulbecco's modified Eagles medium (Nissui, Tokyo, Japan) containing 10\% fetal bovine serum (HiClone). Double strand RNA oligonucleotides targeting the following cDNA sequences were synthesized by Sigma Aldrich: 5'UUCUCCGAACGUGUCACGUTT-3' for control siRNA, 5'-CUUCACGUCGUGAAUACCUCA-3' for CNOT1 \#1 SiRNA, 5'-CAAUUCGCCA ACUUAUCAUGC-3' for CNOT1 \#2 SIRNA, 5'-CUAGCAGGACAAAU AGCAUGA-3' for CNOT2 SIRNA, 5'-GGACGACGUUGAGUACUAUGU-3' for CNOT3 siRNA, 5'-GUUCGUCAAGUUAACUUAUGG -3' for CNOT6 siRNA, 5'-GACCCAGAGUAUUCUGAUGTT-3' for CNOT6L SIRNA, 5'-GACUCUAUAGAGCUACUAACA-3' for CNOT7 SiRNA, 5'-GCAUCCUGGCGAUUAUCAACA-3' for CNOT8 siRNA, 5'CAUGCUGUGGCAUUCAUUUGG-3' for CNOT9 SiRNA, and 5'GCUAUCAUCGUAAAAUAGUUU-3' for CNOT10 siRNA. These siRNAs were transfected into HeLa cells and HEK293T cells using Oligofectamine $^{\mathrm{TM}}$ RNAi MAX (Invitrogen). Seventy two hours after transfection, cells were harvested and used for further experiments. Plasmids were transfected into HeLa cells using FuGENE® 6 Transfection Reagent (Roche). HEK293T cells were transfected 
A

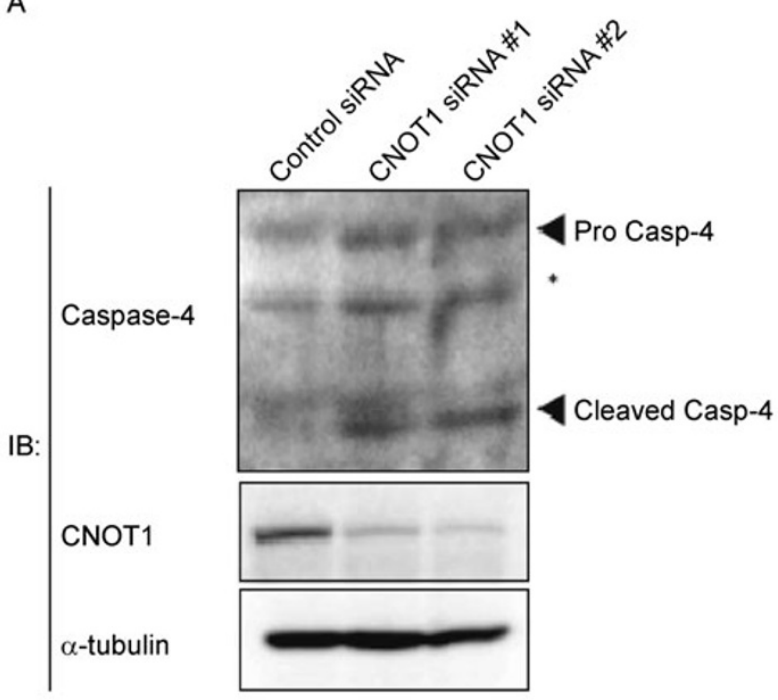

B

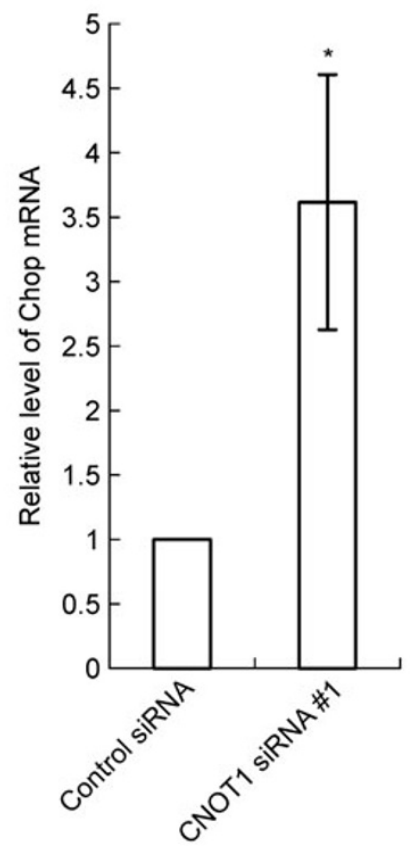

Figure 5. Loss of function caused by CNOT1 depletion leads to ER stress. HeLa cells were transfected with control or CNOT1 siRNA. (A) Seventy-two hours after siRNA transfection, cells were harvested and analyzed by immunoblotting with the indicated antibodies. The positions of pro-caspase-4 (Pro-Casp-4), cleaved caspase-4 (cleaved Casp-4), are indicated with arrowheads. An asterisk shows bands that appear to be derived from pro-caspase-4 by an unknown processing reaction (Hitomi et al., 2004). (B) Forty-eight hours after siRNA transfection, cells were harvested and their total RNA was extracted. The total RNA was reverse-transcribed into complementary DNA using oligo-dT primers. The $\mathrm{CHOP}$ cDNA level was determined by quantitative RT-PCR. Error bars represent the SE of three independent experiments. *: $p<0.05$, Student's $t$-test for control siRNA versus CNOT1 SiRNA. with $10 \mathrm{~g}$ of total DNA by calcium-phosphate transfection method. Sixty six hours after siRNA transfection, the culture medium was replaced with the media containing $10 \mathrm{~mol} / \mathrm{L}$ MG132 and the cells were cultured for another $6 \mathrm{~h}$ before harvest.

\section{Deadenylase assay}

HEK293T cells were transfected with CNOT1 siRNAs. Twenty-four hours after siRNA transfection, cells were transfected with EGFPCNOT6L expression plasmids. After another $24 \mathrm{~h}$, lysates were prepared from the cells and immunoprecipitated with an anti-GFP antibody. The precipitates were incubated at $37^{\circ} \mathrm{C}$ for $180 \mathrm{~min}$ with synthesized RNA substrate (5'-UCUAAAUAAAAAAAAAAAAA AAAAAAA-3'; final concentration, $0.1 \mu \mathrm{mol} / \mathrm{L}$ ) labeled with fluorescein isothiocyanate at the $5^{\prime}$ end. The reaction products were fractionated on a $7 \mathrm{~mol} / \mathrm{L}$ urea- $25 \%$ polyacrylamide denaturing gel. The gel was analyzed with an FLA-5000 Fluorescence Imager (Fujifilm).

\section{Immunoprecipitation and immunoblotting}

Cells were lysed with $1 \%$ NP-40 TNE buffer $[50 \mathrm{mmol} / \mathrm{L}$ Tris- $\mathrm{HCl}(\mathrm{pH}$ 7.5), $1 \mathrm{mmol} / \mathrm{L}$ EDTA, $150 \mathrm{mmol} / \mathrm{L} \mathrm{NaCl}, 1 \mathrm{mmol} / \mathrm{L} \mathrm{NaF}$ ] on ice for $15 \mathrm{~min}$ and clarified by centrifuging. The samples were separated by SDS-PAGE and transferred to Immobilon ${ }^{\mathrm{TM}}$ Transfer Membrane (Millipore). Immunoblotting was performed as described previously (Morita et al. 2007).

\section{Flowcytometry analysis}

Seventy two hours after siRNA transfection, the cells were fixed with ethanol and stained with propidium iodide. The cells were analyzed with FACSCalibur (Becton Dickinson).

\section{Immunofluorescence}

HeLa cells were grown on glass cover slips and fixed with $2 \%$ paraformaldehyde/PBS at room temperature for $10 \mathrm{~min}$. Immunofluorescence staining was performed as described previously (Morita et al., 2007). Photomicrographs were obtained using a Fluoview FV1000 confocal laser scanning biological microscope (Olympus).

\section{Quantitative real time RT-PCR}

Total RNA was extracted from cells using ISOGEN (Nippon Gene) and subjected to reverse transcription using Superscript ${ }^{\mathrm{TM}}$ III reverse transcriptase and oligo dT12-18 primers (Invitrogen). Quantitative real-time RT-PCR was then performed using the ABI Prism 7900HT sequence detection system (Applied Biosystems). The primer sets used in this study are as described previously (Lin et al., 2007).

\section{ACKNOWLEDGEMENTS}

This work was supported by grants-in-aid from the Japan Society for the Promotion of Science and from the Ministry of Education, Culture, Sports, Science and Technology, Japan. 


\section{ABBREVIATIONS}

DEDD, Asp-Glu-Asp-Asp; EEP, exonuclease-endonuclease-phosphatase; PARP, poly ADP ribose polymerase; PERK, protein kinase RNA-like ER kinase; siRNAs, small interfering RNAs; TTP, tristetraprolin; UPR, unfolded protein response

\section{REFERENCES}

Bartlam, M., and Yamamoto, T. (2010). The structural basis for deadenylation by the CCR4-NOT complex. Protein Cell 1, 443-452.

Behm-Ansmant, I., Rehwinkel, J., Doerks, T., Stark, A., Bork, P., and Izaurralde, E. (2006). mRNA degradation by miRNAs and GW182 requires both CCR4:NOT deadenylase and DCP1:DCP2 decapping complexes. Genes Dev 20, 1885-1898.

Berthet, C., Morera, A.M., Asensio, M.J., Chauvin, M.A., Morel, A.P., Dijoud, F., Magaud, J.P., Durand, P., and Rouault, J.P. (2004). CCR4-associated factor CAF1 is an essential factor for spermatogenesis. Mol Cell Biol 24, 5808-5820.

Chen, C., Ito, K., Takahashi, A., Suzuki, T., Ge, W., Nakazawa, T., Yamamoto, T., and Yokoyama, K. (2011). Distinct expression patterns of the subunits of the CCR4-NOT deadenylase complex during neural development. Biophys Biochem Res Commun 411, 360-364.

Chicoine, J., Benoit, P., Gamberi, C., Paliouras, M., Simonelig, M., and Lasko, P. (2007). Bicaudal-C recruits CCR4-NOT deadenylase to target mRNAs and regulates oogenesis, cytoskeletal organization, and its own expression. Dev Cell 13, 691-704.

Collart, M.A. (2003). Global control of gene expression in yeast by the Ccr4-Not complex. Gene 313, 1-16.

Collart, M.A., and Timmers, H.T. (2004). The eukaryotic Ccr4-not complex: a regulatory platform integrating mRNA metabolism with cellular signaling pathways? Prog Nucleic Acid Res Mol Biol 77, 289-322.

Eulalio, A., Behm-Ansmant, I., Schweizer, D., and Izaurralde, E. (2007). P-body formation is a consequence, not the cause, of RNAmediated gene silencing. Mol Cell Biol 27, 3970-3981.

Garneau, N.L., Wilusz, J., and Wilusz, C.J. (2007). The highways and byways of mRNA decay. Nat Rev Mol Cell Biol 8, 113-126.

Graces, R., Gillon, W., and Pai, E.F. (2009). Atomic model of human Rcd-1 reveals an armadillo-like-repeat protein with in vitro nucleic acid binding properties. Protein Sci 16, 176-188.

Harding, H.P., Novoa, I., Zhang, Y., Zeng, H., Wek, R., Schapira, M., and Ron, D. (2000). Regulated translation initiation controls stressinduced gene expression in mammalian cells. Mol Cell 6, 1099-1108.

Hitomi, J., Katayama, T., Taniguchi, M., Honda, A., Imaizumi, K., and Tohyama, M. (2004). Apoptosis induced by endoplasmic reticulum stress depends on activation of caspase-3 via caspase- 12 . Neurosci Lett 357, 127-130.

Ito, K., Inoue, T., Yokoyama, K., Morita, M., Suzuki, T., and Yamamoto, T. (2011). CNOT2 depletion disrupts and inhibits the CCR4-NOT deadenylase complex and induces apoptotic cell death. Genes Cells 16, 368-379.

Lin, J. H., Li, H., Yasumura, D., Cohen, H. R., Chao, Z., Panning, B., Shokat, K. M., LaVail, M. M., and Walter, P. (2007). IRE1 signaling affects cell fate during the unfolded protein response. Science 318 , 944-949.

Maillet, L., Tu, C., Hong, Y.K., Shuster, E.O., and Collart, M.A. (2000). The essential function of Not1 lies within the Ccr4-Not complex. J Mol Biol 303, 131-143.

Miyasaka, T., Morita, M., Ito, K., Suzuki, T., Fukuda, H., Takeda, S., Inoue, J., Semba, K., and Yamamoto, T. (2008). Interaction of antiproliferative protein Tob with the CCR4-NOT deadenylase complex. Cancer Sci 99, 755-761.

Morita, M., Suzuki, T., Nakamura, T., Yokoyama, K., Miyasaka, T., and Yamamoto, T. (2007). Depletion of mammalian CCR4b deadenylase triggers elevation of the p27Kip1 mRNA level and impairs cell growth. Mol Cell Biol 27, 4980-4990.

Nakagawa, T., Zhu, H., Morishima, N., Li, E., Xu, J., Yankner, B.A., and Yuan, J. (2000). Caspase-12 mediates endoplasmic-reticulum-specific apoptosis and cytotoxicity by amyloid-beta. Nature 403, 98-103.

Nakamura, T., Yao, R., Ogawa, T., Suzuki, T., Ito, C., Tsunekawa, N., Inoue, K., Ajima, R., Miyasaka, T., Yoshida, Y., et al. (2004). Oligoastheno-teratozoospermia in mice lacking Cnot7, a regulator of retinoid X receptor beta. Nat Genet 36, 528-533.

Neely, G.G., Kuba, K., Cammarato, A., Isobe, K., Amann, S., Zhang, L., Murata, M., Elmén, L., Gupta, V., Arora, S., et al. (2010). A global in vivo Drosophila RNAi screen identifies NOT3 as a conserved regulator of heart function. Cell 141, 142-153.

Sandler, H., Kreth, J., Timmers, H.T.M., and Stoecklin, G. (2011). Not1 mediates recruitment of the deadenylase Caf1 to mRNAs targeted for degradation by tristetraprolin. Nucleic Acids Res 39, 4373-4386.

Schröder, M., and Kaufman, R.J. (2005). ER stress and the unfolded protein response. Mutat Res 569, 29-63.

Sheth, U., and Parker, R. (2003). Decapping and decay of messenger RNA occur in cytoplasmic processing bodies. Science 300 , 805-808.

Temme, C., Zaessinger, S., Meyer, S., Simonelig, M., and Wahle, E. (2004). A complex containing the CCR4 and CAF1 proteins is involved in mRNA deadenylation in Drosophila. EMBO $\mathrm{J} \mathrm{23,}$ 2862-2871.

Temme, C., Zhang, L., Kremmer, E., Ihling, C., Chartier, A., Sinz, A., Simonelig, M., and Wahle, E. (2010). Subunits of the Drosophila CCR4-NOT complex and their roles in mRNA deadenylation. RNA 16, 1356-1370.

Tucker, M., Staples, R.R., Valencia-Sanchez, M.A., Muhlrad, D., and Parker, R. (2002). Ccr4p is the catalytic subunit of a Ccr4p/Pop2p/ Notp mRNA deadenylase complex in Saccharomyces cerevisiae. EMBO J 21, 1427-1436.

Wang, H., Morita, M., Yang, X., Suzuki, T., Yang, W., Wang, J., Ito, K., Wang, Q., Zhao, C., Bartlam, M., et al. (2010). Crystal structure of the human CNOT6L nuclease domain reveals strict poly $(A)$ substrate specificity. EMBO J 29, 2566-2576.

Winkler, G.S., Mulder, K.W., Bardwell, V.J., Kalkhoven, E., and Timmers, H.T. (2006). Human Ccr4-Not complex is a liganddependent repressor of nuclear receptor-mediated transcription. EMBO J 25, 3089-3099.

Zinszner, H., Kuroda, M., Wang, X., Batchvarova, N., Lightfoot, R.T., Remotti, H., Stevens, J.L., and Ron, D. (1998). CHOP is implicated in programmed cell death in response to impaired function of the endoplasmic reticulum. Genes Dev 12, 982-995 